\title{
Un Typhloceras nouveau de Tunisie (Siphonaptera, Hystrichopsyllidae)
}

\author{
par J.-C. BEAUCOURNU * et T. ABDELKHALEK ** \\ (Collaboration technique: H. Hellal ${ }^{* * *}$ ) \\ * Laboratoire de Parasitologie, Faculté de Médecine, F 35000 Rennes. \\ ** Laboratoire de Parasitologie, Faculté de Médecine, Tunis, République tunisienne. \\ *** Direction de la Médecine préventive et sociale ( $\left.\mathrm{P}^{*} \mathrm{NACEF}\right)$, Tunis, République tunisienne.
}

\section{Résumé.}

Typhloceras favosus benrachidi ssp. nova, parasite d'Apodemus sylvaticus ssp., se distingue de tous les autres Typhloceras connus, par la morphologie des segments VIII et IX chez le mâle.

Une éventuelle synonymie avec $T$. $f$. asunicus Jordan, décrit sur une seule femelle, est discutée.

\section{Summary.}

\section{A new Typhloceras from Tunisia.}

Typhloceras favosus benrachidi ssp. nova, parasitic on Apodemus sylvaticus ssp., can be separated from all others Typhloceras, by the morphology of segments VIII and IX in the male.

Synonymy with $T$. f. asunicus Jordan, described on female only, is discuted.

Reçu le 11 mars 1977. 
Au cours d'une prospection dans le nord de la Tunisie, prospection effectuée sur l'initiative du laboratoire de Parasitologie de la Faculté de Médecine de Tunis ( $\mathrm{P}^{\mathrm{r}}$ Ben Rachid), nous avons eu l'occasion de récolter un Typhloceras particulièrement original que nous décrivons ci-après. Typhloceras Wagner 1903 est, par ailleurs, un genre nouveau pour la Tunisie.

La Puce que nous étudions ici se distingue immédiatement des autres Typhloceras, $T$. poppei et $T$. favosus par la morphologie des segments génitaux du mâle. Nous estimons toutefois qu'il y a lieu de la considérer comme une simple sousespèce de $T$. favosus car, d'une part, elle montre le phallosome de cette espèce (celui de $T$. poppei est légèrement différent), d'autre part, certains caractères originaux de sa chaetotaxie sont également observés chez une autre sous-espèce nouvelle en cours de description (Beaucournu et Gosalbez, sous presse), sous-espèce appartenant manifestement à $T$. favosus par la morphologie de ses segments génitaux.

Nous sommes heureux de dédier cette forme nouvelle au $\mathrm{D}^{\mathrm{r}}$ Ben Rachid, Professeur à la Faculté de Médecine de Tunis, en remerciement et en témoignage de cordiale amitié (1).

\section{Matériel de description}

Mâle holotype, femelle allotype et une femelle paratype sur Apodemus sylvaticus ssp., Aïn Draham, N.-O. de la Tunisie, 4 novembre 1976, un mâle et trois femelles paratypes, même hôte et même endroit, 16 et 17 décembre 1976, un mâle et une femelle paratypes, même hôte et même endroit, 13 février 1977.

Dépôt des types dans la collection Beaucournu et dans celles de la Direction de la Médecine Préventive et Sociale à Tunis.

\section{Description}

T. favosus benrachidi se distingue immédiatement des autres sous-espèces dont le mâle est connu [ $T$. $f$. favosus Jordan et Rothschild, $T$. $f$. rolandi Jordan sensu Beaucournu 1974 (2) et $T$. favosus ssp. nova Beaucournu et Gosalbez, sous presse] par la morphologie très particulière du basimère.

Cuticule très réticulée comme il est de règle chez $T$. favosus. Capsule céphalique, thorax, pattes et segments non modifiés comme chez $T$. $f$. rolandi. Ctenidie prothoracique de 22 épines chez les 9 exemplaires récoltés ( 22 en moyenne chez T. f. rolandi; 20,21 et 22 chez les 3 exemplaires connus de $T$. f. favosus); 16 à

(1) Nos remerciements vont également à tous les stagiaires du cours d'Entomologie médicale de la Faculté de Médecine de Tunis, pour leur coopération sur le terrain.

(2) $T$. f. rolandi Jordan a été décrit sur une seule femelle des Pyrénées-Atlantiques (France). Aucun mâle topotype n'étant récolté, nous avons assimilé à cette sous-espèce tous les exemplaires de $T$. favosus que nous avons collectés en France et en Espagne. 


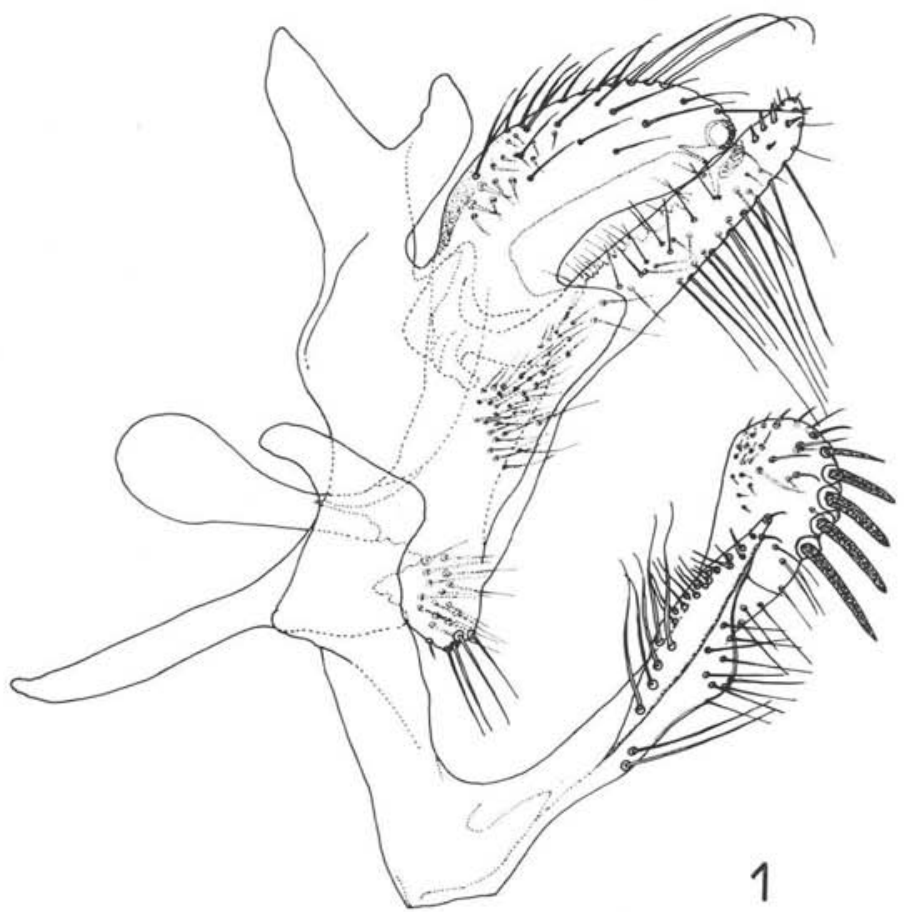

FIG. 1. - T. favosus benrachidi ssp. n., segment IX, mâle holotype.

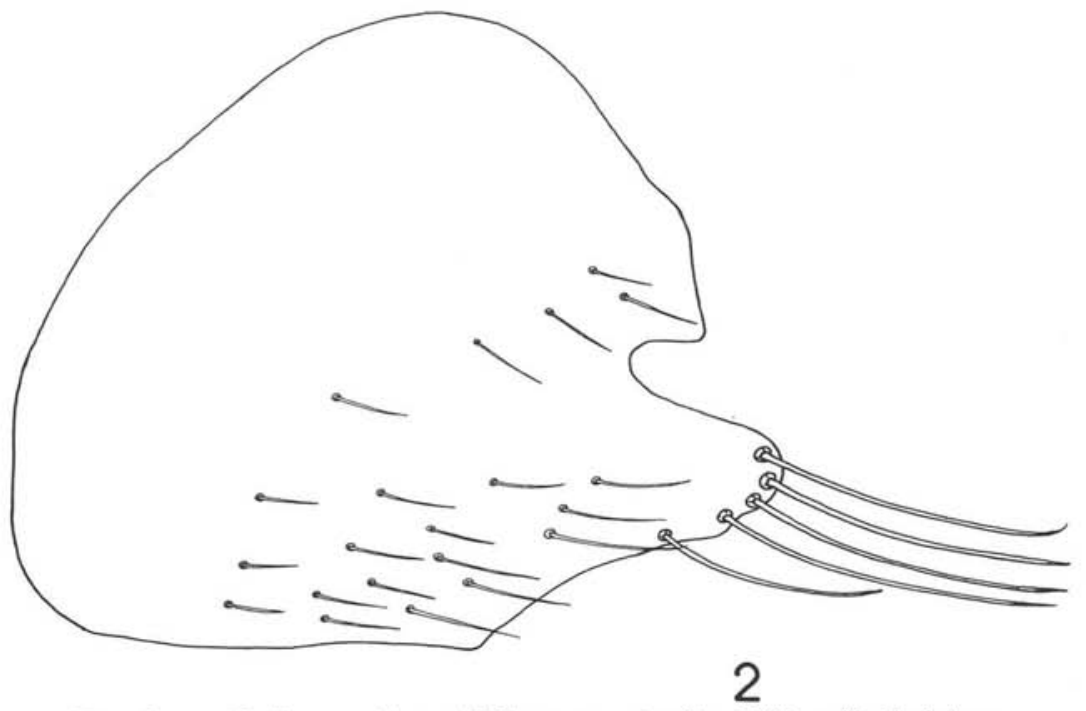

FIG. 2. - T. favosus benrachidi ssp. n., sternite VIII, mâle holotype. 
25 soies sur la face externe du tibia III chez les mâles, 19 à 25 chez les femelles (respectivement 25 et 26,5 en moyenne chez $T$. $f$. rolandi); 10 épines en moyenne de chaque côté sur les tergites abdominaux II à $\mathrm{V}$ comme chez $T$. $f$. rolandi.

Segments modifiés mâles (fig. 1 et 2). Processus basimeris allongé réniforme, le bord dorsal régulièrement convexe ne présentant pas d'angulation postéro-dorsale comme chez les 3 autres sous-espèces dont le mâle est connu (T. f. favosus, T. $f$. rolandi et $T$. favosus ssp. nova Beaucournu et Gosalbez). Bord ventral très convexe laissant un hiatus entre la partie médiane du processus basimeris et processus telomeris. 2 longues soies seulement sur le bord dorsal contre 4 à 5 en général chez les autres sous-espèces. Face interne portant moins de soies que chez $T$. f. rolandi, les plus antérieures spiniformes.

Sternite IX très caractéristique: bras proximal très large; bras distal présentant deux élargissements, l'un dorsal, l'autre ventral, très accentué avec 5 à 9 soies ; 4 à 5 soies spiniformes à son extrémité.

Sternite VIII caractérisé par l'absence de la grande soie insérée au-dessus du sinus et présente chez $T$. poppei, $T$. $f$. favosus et $T$. f. rolandi. Bord postéro-dorsal présentant une angulation nette. Lobe sétigère bien développé, plus allongé que chez T. f. favosus et $T$. f. rolandi.

Segments génitaux femelles (fig. 3) apparemment non distinctifs. Toutefois, une soie interne de l'extrémité postéro-ventrale du tergite VIII est constamment très développée, plus qu'il n'est de règle chez $T$. favosus rolandi.

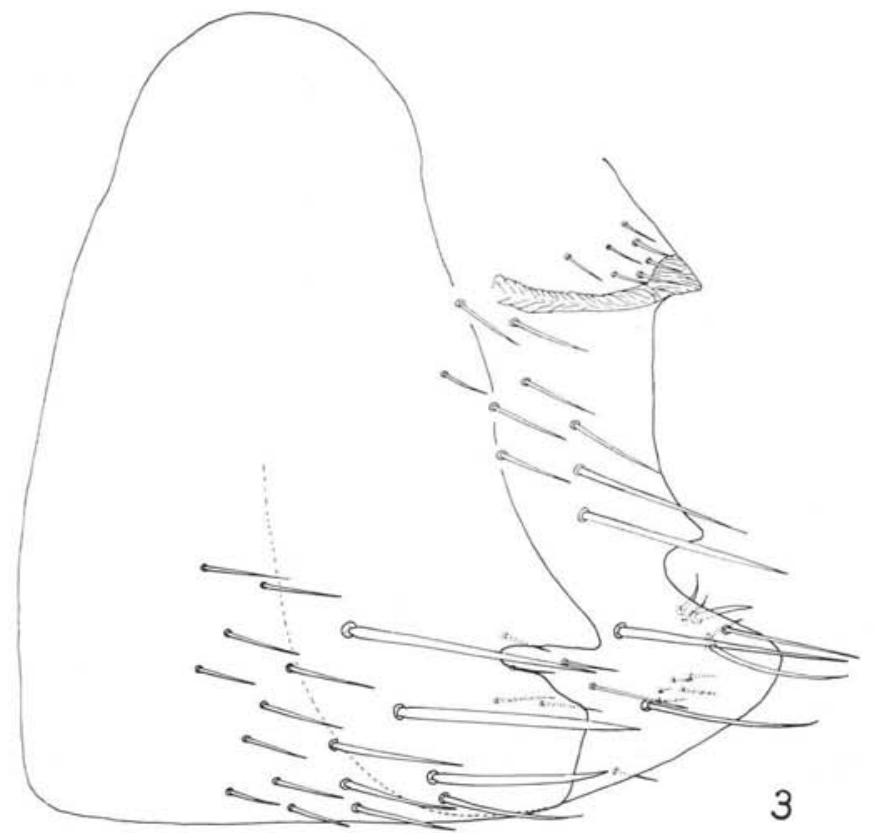

FIG. 3. - T. favosus benrachidi ssp. n., tergite VIII et sternite VII, femelle allotype. 


\section{Discussion}

Si $T$. f. benrachidi s'oppose immédiatement aux autres sous-espèces dont les mâles sont décrits, il subsiste un doute concernant T. $f$. asunicus Jordan, de Sardaigne, seulement connu par la femelle holotype. Nous avons antérieurement montré (1974) que le critère retenu pour distinguer cette sous-espèce est sans valeur. Néanmoins, étant donné les affinités, ou les échanges, faunistiques entre la Tunisie et les Iles méditerranéennes, on ne peut exclure que $T$. $f$. benrachidi et $T$. $f$. asunicus, soit une même sous-espèce, $T$. f. asunicus ayant alors la priorité.

Il est à rappeler, par ailleurs, que nous considérons $T$. favosus comme inféodé, en Europe, à la Musaraigne Crocidura russula (Beaucournu et coll., 1973; Beaucournu 1976). C'est, en particulier, sur cet Insectivore qu'est régulièrement prélevé $T$. f. rolandi et $T$. favosus ssp. nova. Par contre, nos 9 exemplaires de $T$. f. benrachidi proviennent du Mulot Apodemus sylvaticus. Ce Rongeur est l'hôte régulier de $T$. poppei et çà et là, essentiellement en Afrique du Nord, celui de $T$. favosus spp. Des exemplaires de Crocidura russula ont été capturés dans le gite d'Ain Draham. Ils n'hébergeaient que Ctenophthalmus russulae russulae, spécifique de cette Musaraigne et dont c'est la première mention pour la Tunisie.

\section{Bibliographie}

Beaucournu (J.-C.), 1974. - Sur les Typhloceras et Atyphloceras de France (Siphonaptera, Hystrichopsyllidae). Ann. Soc. entomol. Fr. (N.S.), 10, 129-148.

Beaucournu (J.-C.), 1976. - Contribution à l'étude des Siphonaptères de Mammifères du Nord-Ouest de la Région méditerranéenne (France, Italie, Péninsule ibérique). Thèse Doct. ès Sciences, $284 \mathrm{p}$. + cartes, tableaux.

Beaucournu (J.-C.), Gilot (B.) et Véricad (J.-R.), 1973. - Contribution à l'étude des Siphonaptères de la Péninsule ibérique. Eos, 49, 49-78.

Beaucournu (J.-C.) et Gosalbez (J.). - Un Typhloceras nouveau de Catalogne (Siphonaptera, Hystrichopsyllidae). Ann. Parasitol. hum. comp. (sous presse). 